Medical Image Analysis, Volume 69, April 2021, Article number 101956

DOI: $10.1016 /$ j.media.2021.101956

\title{
Angle-closure Assessment in Anterior Segment OCT Images via Deep Learning
}

\author{
HuayingHao a,d, YitianZhao ${ }^{\text {a,d,* }}{ }^{\text {, }}$ Qifeng Yan ${ }^{\text {a,d }}$, Risa Higashita ${ }^{\text {f,c }}$, JiongZhang ${ }^{\mathrm{e}}$, YifanZhao ${ }^{\mathrm{g}}$, YanwuXu ${ }^{\text {a,d }}$, FeiLi ${ }^{\text {b,d }}$, Xiula \\ nZhang ${ }^{\mathrm{b}, \mathrm{d}}$, JiangLiu ${ }^{\mathrm{c}, \mathrm{d}, \mathrm{a}, *}$
}

${ }^{a}$ Cixi Institute of Biomedical Engineering, Ningbo Institute of Materials Technology and Engineering, Chinese Academy of Sciences, Ningbo, China

${ }^{b}$ Zhongshan Ophthalmic Center, State Key Laboratory of Ophthalmology, Sun Yat-sen University, Guangzhou, China

${ }^{c}$ Department of Computer Science and Engineering, Southern University of Science and Technology, Shenzhen, China

${ }^{d}$ Glaucoma Artificial Intelligence Diagnosis and Imaging Analysis Joint Research Lab, Guangzhou E Ningbo, China

${ }^{e}$ Laboratory of Neuro Imaging (LONI), Keck School of Medicine, University of Southern California, Los Angeles, USA

${ }^{f_{T}}$ Tomey Corporation, Nagoya, Japan

${ }^{g}$ School of Aerospace, Transport and Manufacturing, Cranfield University, Bedford, UK

\section{A R T I C LE INFO}

Article history:

Keywords: Angle-closure, glaucoma, anterior chamber angle, AS-OCT, deep learning

\begin{abstract}
A B S T R A C T
Precise characterization and analysis of anterior chamber angle (ACA) are of great importance in facilitating clinical examination and diagnosis of angle-closure disease. Currently, the gold standard for diagnostic angle assessment is observation of ACA by gonioscopy. However, gonioscopy requires direct contact between the gonioscope and patients' eye, which is uncomfortable for patients and may deform the ACA, leading to false results. To this end, in this paper, we explore a potential way for grading ACAs into open-, appositional- and synechial angles by Anterior Segment Optical Coherence Tomography (AS-OCT), rather than the conventional gonioscopic examination.The proposed classification schema can be beneficial to clinicians who seek to better understand the progression of the spectrum of angle-closure disease types, so as to further assist the assessment and required treatment at different stages of angle-closure disease. To be more specific, we first use an image alignment method to generate sequences of AS-OCT images. The ACA region is then localized automatically by segmenting an important biomarker - the iris - as this is a primary structural cue in identifying angle-closure disease. Finally, the AS-OCT images acquired in both dark and bright illumination conditions are fed into our Multi-Sequence Deep Network (MSDN) architecture, in which a convolutional neural network (CNN) module is applied to extract feature representations, and a novel ConvLSTM-TC module is employed to study the spatial state of these representations. In addition, a novel time-weighted cross-entropy loss (TC) is proposed to optimize the output of the ConvLSTM, and the extracted features are further aggregated for the purposes of classification. The proposed method is evaluated across 66 eyes, which include 1,584 AS-OCT sequences, and a total of 16,896 images. The experimental results show that the proposed method outperforms existing state-of-the-art methods in applicability, effectiveness, and accuracy.
\end{abstract}

(c) 2021 Elsevier B. V. All rights reserved.

*Corresponding authors: yitian.zhao@nimte.ac.cn; liuj@ sustech.edu.cn 


\section{Introduction}

Glaucoma is one of the leading causes of irreversible blindness worldwide (Tham et al., 2014), and by the identification of gonioscopy accompanied by biometric examination, cases of glaucoma may be classified into two subtypes: primary open angle glaucoma (POAG), and primary angle-closure glaucoma (PACG) (Sawaguchi et al., 2012). PACG is the most severe stage of primary angle-closure disease (PACD), and PACG in particular is a major cause of blindness in Asian populations (Foster and Johnson, 2001).

Drawing on epidemiological research (Foster et al., 2002), clinicians have suggested that PACD may be further divided into different sub-stages: primary angle closure suspect (PACS), primary angle-closure (PAC), and PACG. The common representation of PACD is contacting between trabecular meshwork and peripheral iris. PACS is appositional but nonadhesive angle-closure, which does not permanently contact yet, as shown in Fig. 1 (c-1). In contrast, PAC and PACG are adhesive angle-closure, which represents peripheral anterior synechial contact (synechial angle-closure, PAS), leading to an occludable anterior chamber angle (ACA) with high intraocular pressure (Foster et al., 2004), as shown in Fig. 1 (c-2), while PACG has optical neuropathy. Interventions such as laser peripheral iridotomy and lens extraction can alleviate angle-closure at PACS, the earliest stage of PACD, to reduce the risk of progressing to PAS, and to remain the aqueous humor outflow function and vision loss (Shang et al., 2019). The detection of synechiae are crucial because they upgrade the diagnosis of PACS to PAC, a clinical state that is at higher risk for PACG. On the other hand, the secondary level of grading (PACS and PAC/PACG) will benefit clinicians in better understanding the progression of the spectrum of angle-closure disease types. Therefore, distinguishing appositional angleclosure from synechial angle-closure is primarily important for clinicians to formulate a plan for further treatment.

Currently, the gold standard for diagnostic angle assessment is the observation of ACA by manual gonioscopy. Careful gonioscopy accompanied by biometric examination can lead

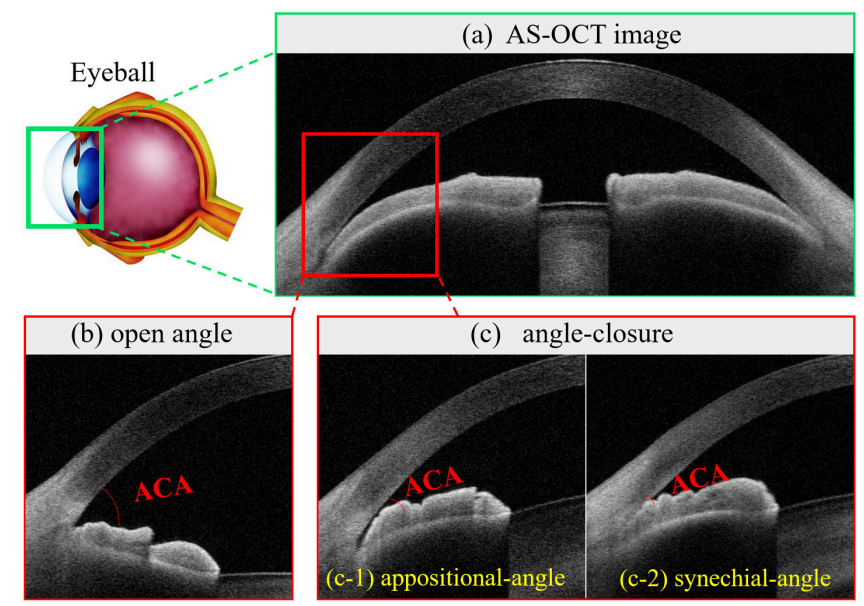

Fig. 1: Illustration of different types of angle-closure disease. (a) A sample ASOCT image: the selected region indicates the region of interest for ACA classification; (b) open-angle; (c-1) appositional-angle; (c-2) synechial-angle. Note, the diagnosis of angle-closure disease usually be made based on gonioscopy examination, we here use AS-OCT images for illustration purpose.

to the correct diagnosis and individualized management in these cases. Fig. 2 (a) shows normal structures seen under gonioscopy. Gonioscopy has two type examinations, staticand dynamic- gonioscopic ones. Static gonioscopy examines whether the angle is occludable or not without the exposure of light and any pressure on the eye (He et al., 2006). Dynamic examination, by contrast, refers to the deliberate application of a controlled degree of pressure and exposed light power to distinguish the appositional and synechial angle status (Casson et al., 2007). Ophthalmologists move the gonioscope counterclockwise, making an annotation every $15^{\circ}$. This annotation accurately describes degree of closure of the ACA in this $15^{\circ}$ region (Matsuo et al., 2020). An example is shown in the left row of Fig. 2 (c), where the red and blue radiant regions indicate that the manual annotation of these ACAs are synechial and appositional angle-closure, respectively, for instances, while the remaining ACAs are open-angle. However, gonioscopy is subjective and relies on the examiner's experience and also timeconsuming. Gonioscopy requires direct contact on the patient's eye, which is uncomfortable for the patient and may deform the ACA, leading to false results.

Anterior segment optical coherence tomography (AS-OCT) is by contrast a non-contact and non-invasive technology for cross-sectional viewing of the anterior segment structures (Radhakrishnan et al., 2005) such as the iris and the ACA. This 


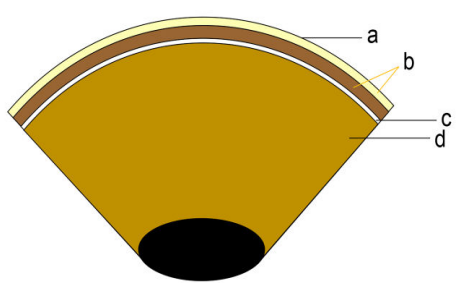

(a)

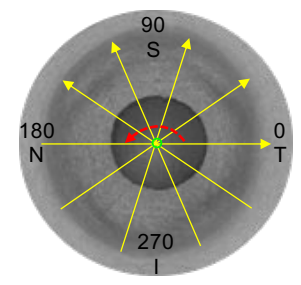

(b)

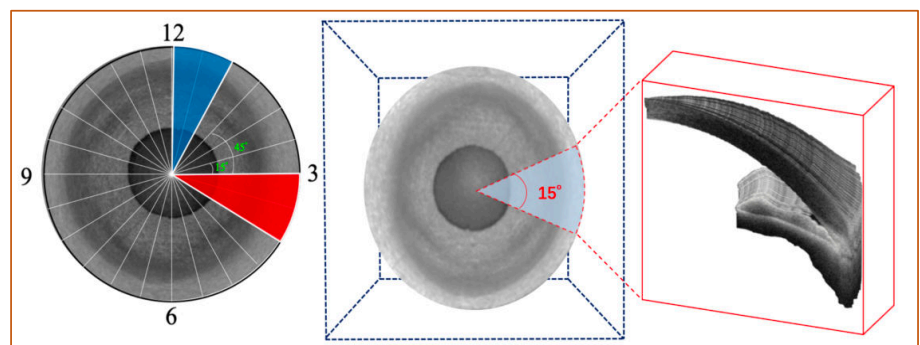

(c)

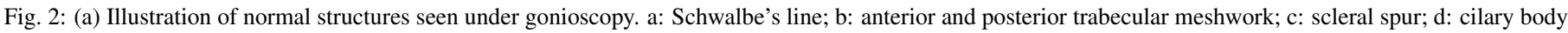

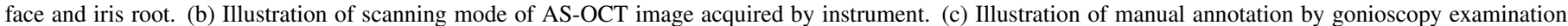

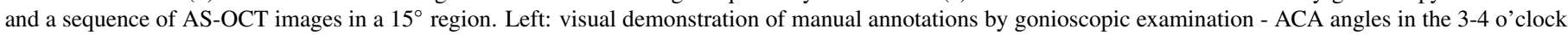

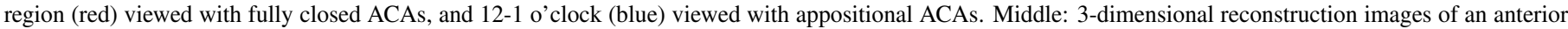
chamber. Right: a sequence of AS-OCT images in a $15^{\circ}$ region, and the alignment of these images to produce a $3 \mathrm{D}$ visualization.

is shown in Fig. 1 (a). Figs. 1 (b) and (c) demonstrate an ACA with open angle, occudable (appositional- and synechial) angle-closure, respectively. Nevertheless, manual annotations based either on gonioscopy or AS-OCT is a tedious task and vulnerable to human errors (Xu et al., 2019). The automated classification of glaucoma subtypes into open angle and angleclosure using AS-OCT has been studied in recent years, and an accurate assessment of angle anatomy could provide a rapid diagnostic tool to guide clinical management at different stages of glaucoma (Fu et al., 2019). For example, as evidenced by the leader board of a recent grand-challenge - Angle closure Glaucoma Evaluation Challenge (AGE) ${ }^{1}$ (Fu et al., 2020) - the task of open angle and angle-closure classification by means of ASOCT has attained a remarkably high standard of performance by using state-of-the-art deep learning-based classification networks, with a total of 4800 AS-OCT images (1600 training images, 1600 validation images, and 1600 testing images). And an area under ROC curve (AUC) score over the AGE dataset are higher than 0.98, when compared with gonioscopy annotation.

However, all existing automatic methods based on AS-OCT make use of the binary classification of open angle and angleclosure (see Section 2 for literature review), automated classification of open-angle, appositional-angle, and synechiae based on AS-OCT imaging has rarely been explored, despite its potential significance in understanding disease progression. This is because it is difficult to distinguish appositional angle from fully closed angle based solely on a single static AS-OCT image. In this work, we intend to explore a potential way for the grading of ACAs into open-, appositional-, and synechialangles by AS-OCT imagery, rather than the conventional gonioscopic examination, so as to guide the classification of POAG, PACS and PAC/PACG.

Inspired by the example of the dynamic gonioscopy examination, we introduce a Multi-Sequence Deep Network (MSDN), which learns to identify discriminative representations from a sequence of AS-OCT images, especially with a view to improving performance in separating appositional-angle from its occludable angle forms. By means of AS-OCT imaging technology, such as provided by the CASIA-2 AS-OCT machine (Tomey Inc., Japan), which scans counter clockwise from the $180^{\circ}-0^{\circ}$ cross-section, as shown in Fig. 2 (b). This technique captures a series of sequence of AS-OCT images. A total of 128 cross-sections are obtained by repeated $1.4^{\circ}$ interval scanning so that eleven continuous scans are captured in a $15^{\circ}$ region. We can analyze this portion of the complete 3D volume as a sequence of $2 \mathrm{D}$ images. The center image of Fig. 2 (c) illustrates a $3 \mathrm{D}$ reconstruction image of an anterior chamber, while the final image of Fig. 2 (c) shows the AS-OCT image sequence in this $15^{\circ}$ region. (It should be noted that we modified these AS-OCT images to improve 3D visualization of the anterior structures, by such procedures as background removal and alignment.)

This work is a substantial extension to our previous

\footnotetext{
${ }^{1}$ https://age.grand-challenge.org/
} 
work (Hao et al., 2019) where features extracted from only a single image were used for angle-closure disease classification. In this work, we have developed a deep learning based method for discriminating the spatial state of features from ASOCT image sequences, rather than from a single image. To our knowledge, this work is the first attempt to classify ACAs into open, appositional- and synechial- angle-closure, using ASOCT data. In addition, we have constructed a AS-OCT dataset for which the AS-OCT of each eye were acquired under both dark and bright illumination conditions. Imaging of each patients was performed first in dark condition, and then under bright condition using a measured standardized light source: dark (0.4 lux) and bright (104 lux) illumination. We take advantage of the resulting changes in pupil size to simulate the pressure of the goniolens, which can push the angle open and help determine the true angle configuration.

\section{Related work}

In the last decade, various approaches have been proposed to the identification of open angle and angle-closure disease from AS-OCT imaging automatically, based on the representations of ACA.

Console et al. (Console et al., 2008) introduced a semiautomated system to produce various anterior segment clinical measurements. It requires the user to identify the position of the scleral spurs, making it unable to form the basis of automatic analysis on a lager-scale dataset. Tian et al. (Tian et al., 2011) provided a segmentation, edge detection and linear regressionbased approach for High-Definition OCT (HD-OCT) to compute ACA measurements. Ni et al. (Ni Ni et al., 2014) assessed the angle structure by mean of continuous measurement of the angle opening distance centered on the Schwalbe's line and the area of the anterior chamber on swept source OCT images. Xu et al. first localized the ACA region, and then classified ACA into open angle and angle-closure-based visual features in the AS-OCT images (Xu et al., 2012), (Xu et al., 2013). Fu et al. (Fu et al., 2017) proposed a data-driven approach which is integrated by AS-OCT segmentation, clinical parameters measurement, and glaucoma screening. Amil et al. (Amil et al., 2019) proposed a distance measure method for ordering and classifying anterior chamber optical coherence tomography (OCT) images.

Based on advances in discriminative representations and large-scale data analysis, deep learning has recently demonstrated its superiority in distinguishing open angle from angleclosure. For example, Convolutional Neural Networks (CNNs) have led to improved performance in medical image classification (Krizhevsky et al., 2012; Simonyan and Zisserman, 2014; He et al., 2016), medical image segmentation (Zheng et al., 2015; Badrinarayanan et al., 2017; Gu et al., 2019) and object detection (Girshick et al., 2014; Redmon et al., 2016; Girshick, 2015; Ren et al., 2015).

For anterior chamber angles classification, there have also been several recent works (Fu et al., 2018; Xu et al., 2019) achieving promising performance for glaucoma type classification in AS-OCT images, as deep learning techniques have learned discriminative representations directly from the images. In (Fu et al., 2018), Fu et al. proposed a Multi-Context Deep Network architecture, in which parallel Convolutional Neural Networks are applied to ACA regions and corresponding scales that are known to be informative for clinically diagnosing angle-closure glaucoma. Fu et al. (Fu et al., 2019) further introduced a Multi-Level Deep Network, which includes multiple parallel sub-networks to learn multi-level representations from the multiple regions known to be informative for angleclosure detection in each AS-OCT image. Xu et al. (Xu et al., 2019) employed deep learning classifiers for automated detection of gonioscopic angle closure and primary angle closure disease (PACD). In our previous work (Hao et al., 2019), we proposed a multi-scale regions convolutional neural networks for glaucoma type classification of POAG, PACS and PACG, which include three parallel convolutional neural networks to extract feature representations, and utilize a weighted ensemble to stack feature representations for classification.

\section{Methodology}

In this section, we detail the proposed classification framework. We first utilize a global B-scan alignment method to align 


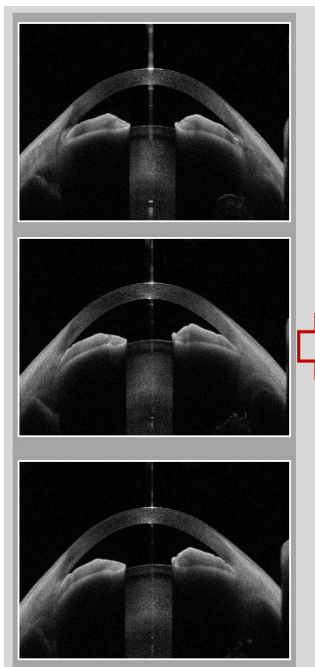

(a) Raw sequence
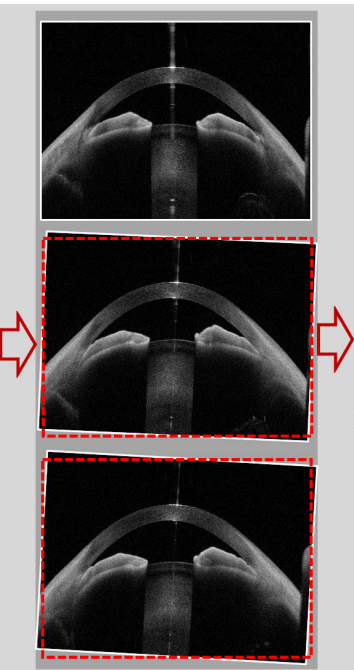

(b) Image alignment
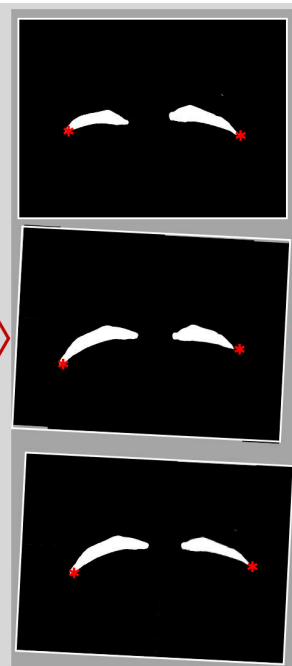

(c) Iris segmentation

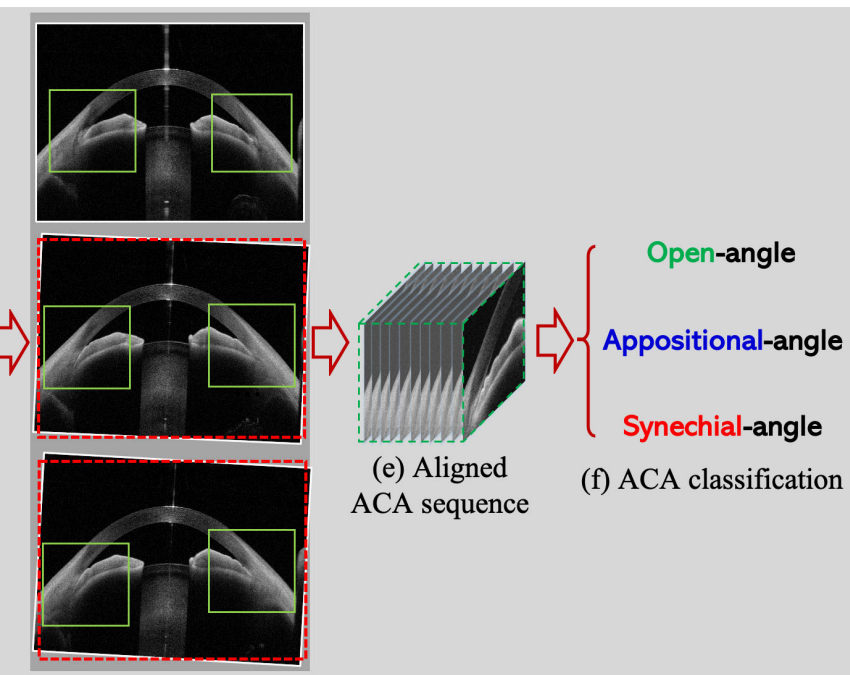

(d) ACA detection

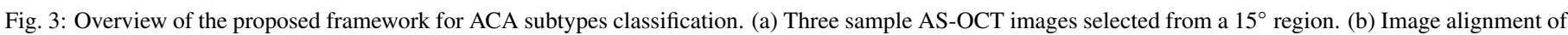

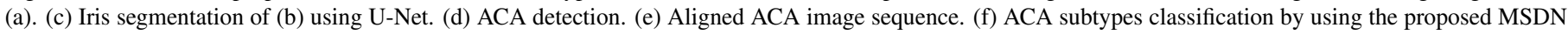
network.

the AS-OCT scans, and then localize ACA by iris segmentation, where the iris could provide the primary structural image cue; finally, our MSDN classified the ACA types by learning the spatial representations from AS-OCT data acquired under two different illumination conditions (bright and dark). The pupil exhibits different responses under various illumination conditions, and the changing of the pupil size may stretch the iris and lead to the morphological changes of ACA. It may clearly be seen that the appositional-angle presents different appearances when the pupil size changes, while synechiae maintains a relatively consistent appearance. In consequence, discriminative representations may be derived from both local appearance features and global geometry under various illumination conditions. Fig. 3 illustrates the overview of the proposed framework.

\subsection{Image alignment}

The possibility of involuntary eye movement and improper placement of the optical axis of the eye could cause misalignment between $2 \mathrm{D}$ scans, which will lead to the resulting image sequence being unreliable (Williams et al., 2016). To this end, an image alignment method is required to align these scans so as to improve the accuracy of subsequent processes.
In this framework, a global AS-OCT alignment is used to overcome the misalignment. Denote a translation $(\Delta x, \Delta y)$ includes both vertical and horizontal directions, and is applied to all consecutive OCT scans. For instance, if $B_{i}$ is the reference scan, $B_{i}$ is aligned to its prior B-scan $B_{i-1}$, and the alignment $\left(\Delta x_{i}, \Delta y_{i}\right)$ can be checked between the scans $B_{i}$ and $B_{i-1}$, so as to minimize their difference (Cheng et al., 2016). A full search of alignment may also be applied, but is time-consuming. Diamond pattern algorithm is proposed by (Zhu and Ma, 2000) to solve motion offset between image sequences. It not only achieves good performance in small-motion and large-motion content datasets, but also requires less computation and is easy to implement compared with other alignment methods. In addition, the diamond search strategy is employed by (Cheng et al., 2016) to OCT image alignment. It is appropriate to apply the diamond search strategy for AS-OCT image alignment. Image alignment based on the diamond search algorithm is carried out in each $15^{\circ}$ region AS-OCT image sequence. Since an ASOCT slice is captured by the instrument per $0.02 \mathrm{~s}$, the angle offset caused by the eye axis offset in the $15^{\circ}$ region is relatively subtle. Therefore, considering the algorithm complexity and the influence of position deviation on LSTM, we employ the diamond search algorithm for translation alignment without 
rotation alignment of AS-OCT sequences. The diamond search strategy iteratively searches for the alignment between $B_{i}$ and $B_{i-1}$ from neighbor points, until the difference between $B_{i}$ and $B_{i-1}$ reaches a stable value. To be more specific, assuming an alignment $(\Delta x, \Delta y)$ which minimizes the objective function as follows:

$$
\min _{\Delta x, \Delta y} \sum_{x, y}\left|B_{i}(x, y)-B_{i-1}(x+\Delta x, y+\Delta y)\right|
$$

In line with the work proposed in (Cheng et al., 2016), two searching patterns are used by the diamond search algorithm. The first pattern is a large diamond search pattern (LDSP), which consists of 9 checking points forming a diamond shape. The second pattern is a small diamond search pattern (SDSP) which consists of 5 checking points, also forming a diamond shape. LDSP is firstly applied until the difference between $B_{i}$ and $B_{i-1}$ reaches a minimum value. Then SDSP is used to determine the best matching between $B_{i}$ and $B_{i-1}$ i.e., the position of minimum difference. Fig. 3 (b) demonstrates examples of image alignment from a randomly selected image sequences.

\subsection{Iris segmentation and ACA detection}

ACA region localization is an important step for discriminative feature learning in the subsequent deep learning network. Various methods have been proposed to detect the ACA region by utilizing geometric properties. Xu et al. (Xu et al., 2012) proposed a geometric method for ACA region detection based on edge detection and shape properties. Tian et al. (Tian et al., 2011) detected the ACA in High-Definition OCT (HD-OCT) images by localizing of the Schwalbe's line. Fu et al. (Fu et al., 2017) proposed an automated AS-OCT structure segmentation approach, which transfers manually marked labels to the target image for guiding segmentation, so as to determine the ACA region. However, the quality of images will greatly affect the performance of these methods (Xu et al., 2012) (Tian et al., 2011). The pixel segmentation method (Fu et al., 2017) is obviously more accurate than coarse ACA localization and clinical parameter measurement, but it is time consuming.

In this work we propose an effective ACA localization method by accurately segmenting the iris from AS-OCT using

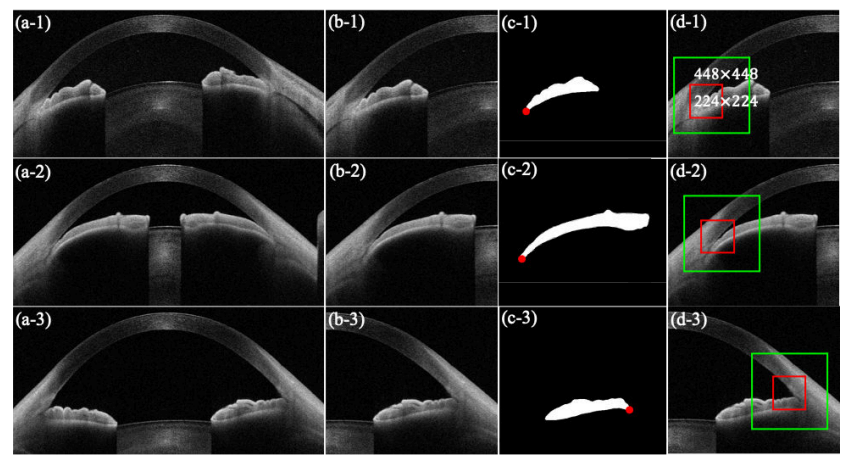

Fig. 4: Outline of the preprocessing steps. (a-1)-(a-3): Input AS-OCT images. (b-1)-(b-3): Split images. (c-1)-(c-3): Iris segmentation by U-Net, and the red dot indicates the iris root. (d-1)-(d-3): ACA regions with two different scales. Red and green bounding boxes are of sizes $224 \times 224$ and $448 \times 448$, respectively.

on a deep learning algorithm. The iris root is a salient landmark to locate the coarse ACA region, and in this work we use the iris root as the centroid of the ACA region (with two different sizes of bounding box). It should be noted that, we constructed a sub-dataset for the iris segmentation task: a total of $100 \mathrm{AS}$ OCT images from an aligned image sequence were selected to compose this dataset. As there are two irises in each AS-OCT image, we split each compound image into two distinct iris images, as shown in Fig. 4 (b-1) yielding a total of 200 split iris images, of which 40 images are examples of open angles and 160 images contain instances of appositional and fully closed angles. We further asked two graders to annotate the iris regions of each image manually. Iris contours were firstly delineated and the contours were filled to obtain the iris regions as pixel level annotation. Two annotators formulated two consistency principles before annotation: 1) keep the iris region intact according to the iris edge in AS-OCT image, and 2) determine the unclear iris root contour according to the previous and subsequent slices in the volume. Finally, a consensus between the two graders was used as the reference standard. It is worth noting that in practice, the inter-annotator agreement is higher than 0.90 in terms of pixel-level, and no image was discarded in this 100 AS-OCT subset.

The fully automatic iris segmentation is carried out by a UNet-based convolutional neural network (Ronneberger et al., 2015). This network is made up of a contracting-encoder and an expanding-decoder part, which permits the user to obtain a label 


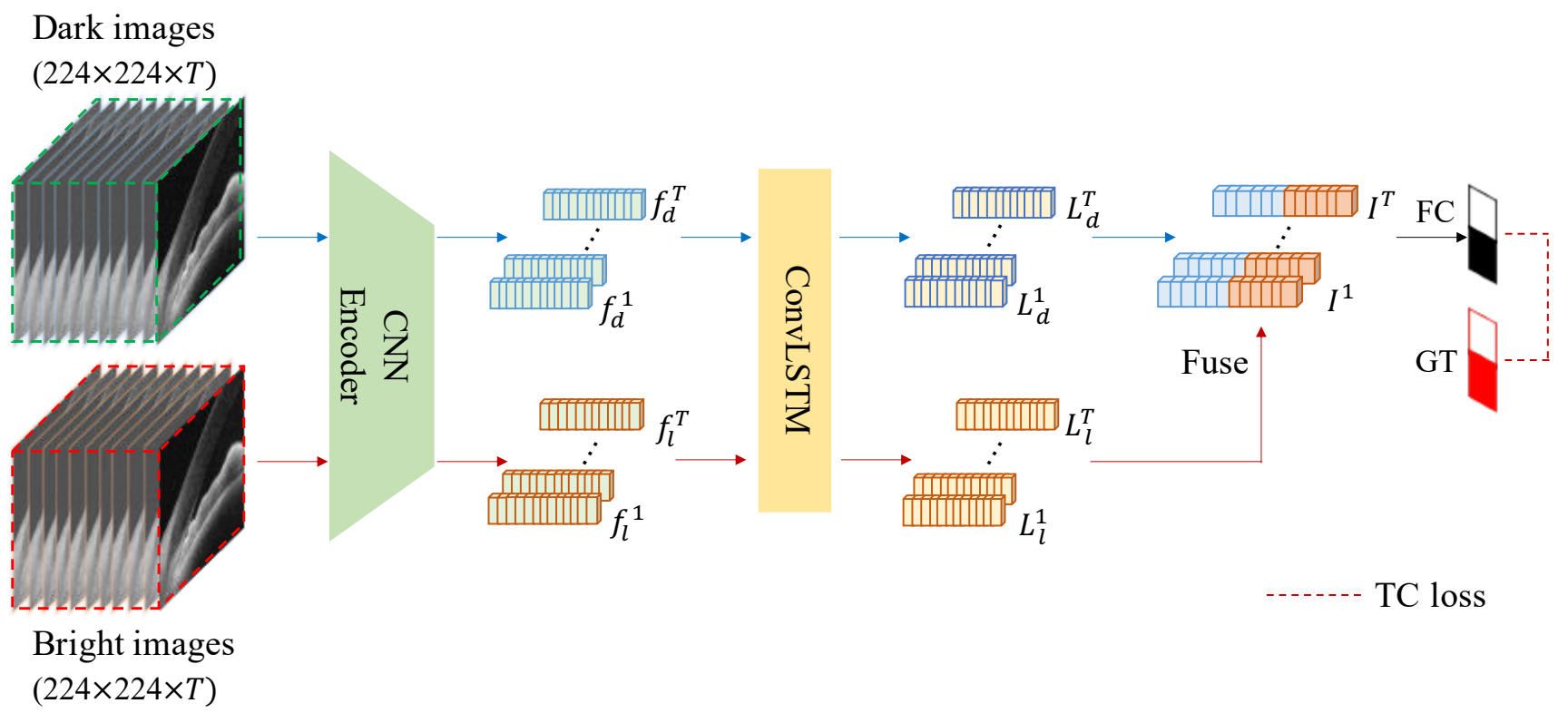

Fig. 5: Architecture of our MSDN for anterior chamber angles classification on Dark \& Bright dataset.

classification for every single pixel. We train the U-Net on an iris image set comprising a randomly sampled $80 \%$ of our full iris image set, leaving out $20 \%$ of this dataset as a testing set. We use a 5-fold method to train the model in order to achieve the best results, and the Mean Square Error (MSE) loss is used. The resulting iris segmentation performance attain an accuracy of 0.99. However, we will not provide qualitative and quantitative evaluations of iris segmentation in this paper, as it is not the main contribution of this work. Fig. 4 (c) demonstrates an iris segmentation performance.

Finally, the ACA region may be determined by the position of the iris root (Fig. 4 (c)), where the iris root may be localized by the left- or rightmost pixel of the segmented iris region. In the experimental work, we obtained two scales of ACA region (Fig. 4 (d)): two bounding boxes with sizes of $224 \times 224$ and $448 \times 448$, centered at the iris root. The effectiveness of the size of ACA regions will be discussed in Section 5.2.

\subsection{ACA classification}

By learning discriminative representations from an AS-OCT image sequence, a deep learning based method is capable of simultaneously modeling both motion and appearance changes, thereby improving performance in separating appositional angle and fully closed angle. In consequence, we introduce a Multi-Sequence Deep Network (MSDN) for ACA subtypes classification. The proposed MSDN consists of two subnetworks to generate representations for ACA subtypes classification: a Convolutional Neural Network (CNN) encoder module and a modified Convolutional Long Short Term Memory (ConvLSTM) module. This is in light of the success of CNN for image representation (Krizhevsky et al., 2012) and of LSTM (Greff et al., 2016) for extraction of changes in sequential data.

The network architecture of our MSDN is shown in Fig. 5. Two streams of aligned ACA images are fed to our MSDN model to obtain a joint representation, combining images captured in both dark and bright illumination environments (see more details in the Section 4.1). Then a multi-layer ConvLSTM is employed to explore the spatial state of features in the last output layer of the CNN Encoder module. Finally, dynamic features from the dark and bright datasets are aggregated for glaucoma classification.

CNN encoder module. Taking into consideration the problems of overfitting and parameter cost, we employ ResNet34 (Fu et al., 2018) in the feature encoder module as our baseline. This retains the first four feature extracting blocks, without the average pooling layer and the fully connected layers. ResNet has a shortcut mechanism to prevent the gradient vanishing and accelerate the network convergence. The in- 


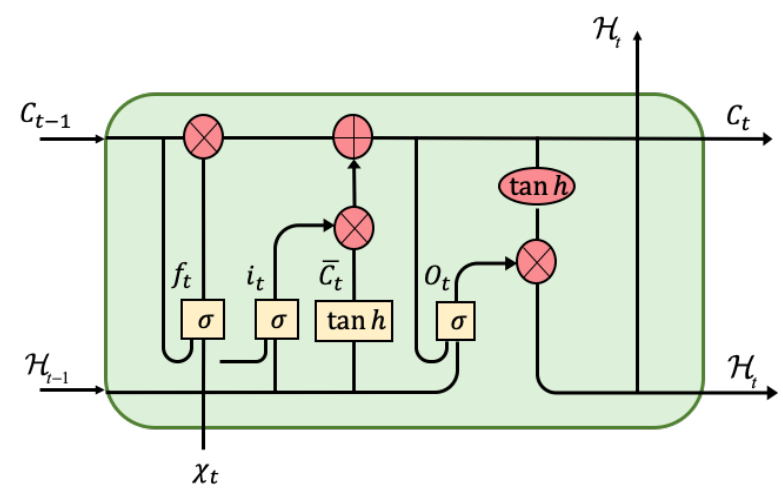

Fig. 6: Illustration of the ConvLSTM unit architecture.

put sequential data to the $\mathrm{CNN}$ encoder module are resized to $224 \times 224$ pixels, taken from the same patient under two different illumination conditions.

ConvLSTM-TC module. LSTM has demonstrated its superiority in modeling motion features for anomaly detection and activity recognition (Malhotra et al., 2016). Recently, a novel ConvLSTM (Xingjian et al., 2015) has been proposed to facilitate data reconstruction by preserving the spatial information. In this paper, we first process the AS-OCT sequence into a special time sequence, and we then employ the ConvLSTM to explicitly deal with sequential AS-OCT images, and propose a new loss, Time-weighted Cross-entropy loss (TC loss), to encode appearance and change of appearance motion.

The formulations of the ConvLSTM may be defined as:

$$
\begin{aligned}
i_{t} & =\sigma\left(W_{x i} * \mathcal{X}_{t}+W_{h i} * \mathcal{H}_{t-1}+W_{c i} \circ C_{t-1}+b_{i}\right), \\
f_{t} & =\sigma\left(W_{x f} * \mathcal{X}_{t}+W_{h f} * \mathcal{H}_{t-1}+W_{c f} \circ C_{t-1}+b_{f}\right), \\
C_{t} & =f_{t} \circ C_{t-1}+i_{t} \circ \tanh \left(W_{x c} * \mathcal{X}_{t}+W_{h c} * \mathcal{H}_{t-1}+b_{c}\right), \\
o_{t} & =\sigma\left(W_{x o} * \mathcal{X}_{t}+W_{h o} * \mathcal{H}_{t-1}+W_{c o} \circ C_{t}+b_{o}\right), \\
\mathcal{H}_{t} & =o_{t} \circ \tanh \left(C_{t}\right)
\end{aligned}
$$

where $\mathcal{X}_{t}$ and $\mathcal{H}_{t}$ are the input and output of the ConvLSTMTC at time step $t$ ( $t$ indicates the $t^{t h}$ frame in a image sequence), and $i_{t}, f_{t}$ and $o_{t}$ indicate the input, forget and output gates, respectively. A memory cell $C_{t}$ stores the historical information. * represents the convolution operation, o denotes the elementwise multiplication, and $\sigma$ is the sigmoid activation function. $W_{m n}$ denotes the weight of convolution function and $b_{n}$ denotes bias, where $\mathrm{m}$ and $\mathrm{n}$ represent input and output of the convolution layer, respectively. Fig. 6 shows the ConvLSTM unit architecture.

As illustrated in Fig. 5, the encoded features of each frame from the dark and bright illumination image sequences are fed into separate layers of a 2-layer ConvLSTM-TC, which memorizes all historic frames. In order to maintain consistency with clinical diagnostic outcome, we integrate the outputs of the ConvLSTM-TC and use a $1 \times 1$ convolution kernel to fuse the features, so as to reduce the dimension of weights and computational cost. We define $L_{d}$ and $L_{l}$ as the output of the ConvLSTM-TC at time step $t$, which is accumulative after feeding $t$ deep features $f_{d}$ and $f_{l}$ into it:

$$
\begin{aligned}
& L_{d}^{t}=\text { ConvLSTM - TC }\left(f_{d}^{1}, f_{d}^{2}, \ldots, f_{d}^{T}\right) \\
& L_{l}^{t}=\text { ConvLSTM }-\mathrm{TC}\left(f_{l}^{1}, f_{l}^{2}, \ldots, f_{l}^{T}\right)
\end{aligned}
$$

The output $I^{t}$ of feature fusion is connected to the fully connected (FC) layer to classify the glaucoma type.

$$
I^{t}=f\left(L_{d}^{t}, L_{l}^{t}\right)
$$

In our paper, we utilize unidirectional ConvLSTM to deal with long distance dependence in AS-OCT sequences. In order to enhance the memory ability of ConvLSTM for AS-OCT slices at different time steps in the sequence, especially the previous frames of a sequence, we define a new TC loss instead of minimizing the loss on the final time step, which can obtain and optimize all the intermediate outputs of every time step.

Ideally, ConvLSTM is expected to learn that the longer the image sequence and the more classification information it processes then the higher the confidence of classification of the ACA type can be achieved (Zhang et al., 2019). Inspired by the principle, a weighted ensemble method based time step is applied to the TC loss. Therefore, the proposed TC loss is defined as:

$$
\mathcal{L}_{\mathrm{TC}}=\frac{1}{n} \sum_{t=1}^{n} \sum_{k=1}^{K}-w_{t}\left[y_{k} \log \left(C\left(I^{t}\right)_{k}\right)\right]
$$

where $C$ and $k$ denote the classifier and classification label, respectively. $n$ denotes the number of images in a sequence. $C\left(I^{t}\right)_{k}$ indicates the classifier $C$ correctly identifies the final output $I$ at time step $t$, and $y_{k} \in\{0,1,2\}$ are the values of ground truth $(\mathrm{GT})$, and $\mathrm{K}=3$ denotes the total number of label. $w_{t}$ is the weight of each frame in a sequence. Taking into account this 


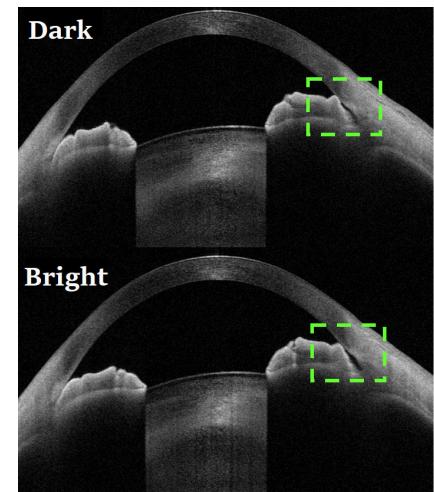

Appositional-angles

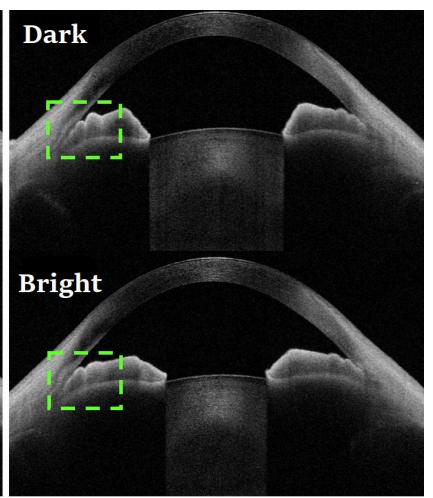

Synechial-angles

Fig. 7: Example AS-OCT images acquired under dark and bright illumination conditions.

TC loss ensures that the features of one AS-OCT image used with the appearance feature of another image can be decoded to the latter AS-OCT scan. In our paper, our sequence length $n$ is 11 and we set $w_{t}=t$ after a series of experiments.

\section{Experiments}

\subsection{Dataset}

A clinical dataset was constructed by Zhongshan Ophthalmic Center, China. All the AS-OCT images were captured along the of the eye's optical axis by a CASIA-2 machine (Tomey Inc., Japan) from 66 eyes of 60 subjects with POAG, PACS or PAC/PACG (annotated by dynamic gonioscopic examinations), and the structure of the anterior chamber appears at a relatively consistent position among the AS-OCT images in practice. In particular, 21 eyes (POAG) are with open ACA, 13 eyes (PACS) are with appositional ACA, and 32 eyes (PAC/PACG) are with appositional ACA and closed ACA images. Each image set contains 128 AS-OCTs, and the resultant image has an area of $2144 \times 1876$ pixels.

It is important to reiterate that this clinical dataset has two sub-sets, referred as Dark and Bright sets in this paper. Fig 7 demonstrates two ACAs under different illumination conditions. We intend to use the resulting changes in pupil size to simulate the pressure by the goniolens. The reason of pupil changes caused by bright and dark environments is the miosis effect (iris dilation) (Hirose et al., 2013), which can open the appositional but non-adhesive anterior chamber angle (ACA) (Aptel et al., 2012). The pressure under gonioscopy also has the same effect. Therefore, we intend to use the resulting changes in pupil size to simulate the pressure by the goniolens, which can push the angle open and help determine the true angle configuration. We raised our hypothesis based on previous studies (Gazzard et al., 2004; Nolan et al., 2007; Lee et al., 2016) indicate morphological change in the iris and anterior chamber between different light conditions.

A senior professor with more than 30 years' experiences on glaucoma diagnosis and analysis made an annotation from gonioscopic examination of on every $15^{\circ}$ ACAs of an eye, yielding 24 annotations for a single eye, resulting in total of 1584 annotations for each dataset. In light of this, we ordered the AS-OCT slices $(T=11$ slices $)$ in each $15^{\circ}$ region into a sequence, generating 24 sequences for each eye, for a total of 1584 image sequences in each of the two Dark and Bright datasets. In particular, 504 sequences were annotated with open ACA, 742 sequences were instances of appositional ACA and 338 sequences present examples of fully closed ACA. Dynamic gonioscopy was performed with corneal indentation. The ophthalmologists imposed pressure on the gonioscopy lens, which can help determine if an angle is anatomically synechial or appositional.

In our experiments, we use 5-fold cross-validation to train and test our model on Dark, Bright and merged datasets at a patient level, which ensures that the ACA images from one patient fall into the same training or test set. In each dataset, we sample at the same category ratio in the subset of the cross-validation experiment.

\subsection{Evaluation Metrics}

Following the standard performance assessment protocol for multi-class classification, we use macro sensitivity (Se), specificity (Sp), accuracy (Acc), and balanced accuracy (B-Acc):

$$
\begin{gathered}
S e=\frac{1}{N_{c}} \sum_{i=1}^{N_{c}} \frac{T P_{i}}{T P_{i}+F N_{i}}, \quad S p=\frac{1}{N_{c}} \sum_{i=1}^{N_{c}} \frac{T N_{i}}{T N_{i}+F P_{i}} \\
A c c=\frac{1}{N_{c}} \sum_{i=1}^{N_{c}} \frac{T P_{i}+T N_{i}}{T P_{i}+F N_{i}+F P_{i}+T N_{i}}, \quad B-A c c=(S e+S p) / 2 .
\end{gathered}
$$

where $\mathrm{TP}_{i}$ indicates true positives, $\mathrm{TN}_{i}$ true negatives, $\mathrm{FP}_{i}$ false positives, $\mathrm{FN}_{i}$ false negatives for the $i$-th classification label. 
H. Hao et al. / Medical Image Analysis (2021)

Table 1: Different classification approaches for the grading of ACAs into open-, appositional-, and synechial-angles on Dark and Bright datasets.

\begin{tabular}{|c|c|c|c|c|c|c|c|c|c|c|c|c|}
\hline & \multicolumn{6}{|c|}{ Dark Dataset } & \multicolumn{6}{|c|}{ Bright Dataset } \\
\hline Methods & kappa & F1 & Acc & B-Acc & $\mathrm{Se}$ & $\mathrm{Sp}$ & Kappa & F1 & Acc & B-Acc & $\mathrm{Se}$ & $\mathrm{Sp}$ \\
\hline $\mathrm{HOG}+\mathrm{SVM}$ & 0.6505 & 0.6748 & 0.8622 & .7849 & 0.6952 & 0.8747 & 6420 & 0.6284 & 0.8611 & 0.7732 & 0.6767 & 0.8698 \\
\hline $\mathrm{HOG}+\mathrm{KNN}$ & 5856 & 0.7134 & 0.8207 & .7921 & 0.7194 & 0.8647 & .5806 & 0.7046 & 0.8207 & 0.7841 & 0.7066 & 0.8616 \\
\hline HOG+AdaBoost & 0.6237 & 0.7242 & 0.8422 & .7984 & 0.7231 & 0.8738 & 0.6295 & 0.7261 & 0.8451 & 0.7999 & 0.7246 & 0.8752 \\
\hline HOG+Random Forest & 0.6696 & 0.7324 & 0.8657 & 0.8075 & 0.7305 & 0.8845 & 0.6557 & 0.7310 & 0.8594 & 0.8065 & 0.7288 & 0.8842 \\
\hline $\mathrm{HOG}+\mathrm{Na}$ & 0.6073 & 0.7266 & 0.8291 & 0.8109 & 0.7455 & 0.8763 & 0.6854 & 0.7752 & 0.8666 & 0.8362 & 0.7765 & 0.8958 \\
\hline MLDN & 749 & 0.7233 & 0.8695 & 3047 & 0.7249 & 8846 & 851 & 0.7377 & 0.8729 & 0.8122 & 0.7358 & 0.8885 \\
\hline CNN & 773 & 0.7498 & 0.8673 & 8171 & 0.7455 & 0.8887 & 156 & 0.7731 & 0.8840 & 0.8328 & 0.7657 & 0.8998 \\
\hline let & 5856 & 0.7522 & 0.8713 & 8191 & 0.7475 & 0.8907 & 0.6949 & 0.7772 & 0.8718 & 0.8364 & 0.7754 & 0.8975 \\
\hline 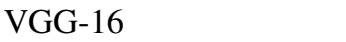 & 012 & 0.7731 & 0.8763 & 8325 & 0.7677 & 0.8974 & 0.6974 & 0.7751 & 0.8738 & 0.8343 & 0.7713 & 0.8973 \\
\hline $\mathrm{R}$ & 5650 & 0.7484 & 0.8632 & 8139 & 0.7401 & 0.8878 & 0.6778 & 0.7786 & 0.8720 & 0.8408 & 0.7806 & 0.9011 \\
\hline & 295 & 0.7941 & 0.8881 & 8468 & 0.7870 & 0.9065 & 0.6951 & 0.7749 & 0.8725 & 0.8343 & 0.7717 & 0.8969 \\
\hline Xcepti & 0.7248 & 0.7834 & 0.8874 & 0.8392 & 0.7749 & 0.9034 & 0.7004 & 0.7805 & 0.8743 & 0.8386 & 0.7781 & 0.8991 \\
\hline $\mathrm{C} 3 \mathrm{D}$ & 489 & 0.8115 & 0.8956 & 8592 & 0.8048 & 9136 & 379 & 0.7992 & 0.8918 & 0.8500 & 0.7911 & 0.9089 \\
\hline I3D & 662 & 0.8171 & 0.9019 & 0.8619 & 0.8073 & 0.9166 & 0.7487 & 0.8038 & 0.8969 & 0.8527 & 0.7938 & 0.9115 \\
\hline S3D & 0.7431 & 0.8007 & 0.8931 & 0.8567 & 0.8016 & 0.9119 & 0.7593 & 0.8154 & 0.9007 & 0.8609 & 0.8059 & 0.9158 \\
\hline $\mathrm{ResNe}$ & 7689 & 0.8281 & 0.9036 & 8714 & 0.8220 & 0.9208 & 0.7920 & 0.8450 & 0.9133 & 0.8829 & 0.8377 & 0.9282 \\
\hline ResNet+Bi-Co & 0.7815 & 0.8319 & 0.9099 & 0.8717 & 0.8206 & 0.9229 & 0.7800 & 0.8294 & 0.9095 & 0.8697 & 0.8175 & 0.9219 \\
\hline MSDN $(224 \times 224)$ & $\mathbf{0 . 7 9 2 0}$ & $\overline{0.8382}$ & 0.9146 & 0.8754 & 0.8250 & 0.9257 & 0.7826 & $\overline{0.8304}$ & 0.9108 & 0.8704 & 0.8181 & $\mathbf{0 . 9 2 2 7}$ \\
\hline
\end{tabular}

In order to reflect the trade-offs between the sensitivity and specificity and evaluate the quality of our classification results more reliably, the kappa analysis ${ }^{2}$ and $\mathrm{F}$-measure $(F 1 \text { score })^{3}$ are further provided. These two measures are more robust than other percentage agreement measure, as they take into account the possibility of the agreement occurring by chance. The kappa values between 0.81 to 1.00 indicate almost perfect agreement, values between 0.61-0.80 exhibit substantial agreement, values of 0.41-0.60 exhibit moderate agreement and values less than 0.40 exhibit poor to fair agreement. $F 1$ score reaches its best value at 1 and worst at 0 .

\subsection{Implementation details}

The proposed method was implemented by the publicly available Pytorch Library. Each stream is fine-tuned from an initialization with the pre-trained ResNet-34 deep model (He et al., 2016) in the CNN Encoder module. The classification part is a stacked 2-layer ConvLSTM, which has 512 hidden units in each of cell. In the training phase, we employed an Adam optimizer to optimize the deep model. We used a gradually decreasing learning rate, starting from 0.0001 , and a mo-

\footnotetext{
${ }^{2}$ https://en.wikipedia.org/wiki/Cohen\%27s_kappa

${ }^{3}$ https://en.wikipedia.org/wiki/F1_score
}

mentum of 0.9. In addition, online data enhancement is employed to enlarge the training sequence data. The same level of data enhancement is used for all images in a sequence, which includes brightness, color, contrast and sharpness transformation, and we set a random seed from 1 to 4 for enhancement.

\subsection{Classification performance}

In this subsection, we report the classification performance over the Dark, Bright, and merged datasets, respectively. In order to demonstrate conclusively the superiority of the proposed method over the the following state-of-the-art methods: (1) 2D deep models with 2D input: conventional classifiers, 2D deep learning networks, and those models are specifically designed for ACA classification; (2) 3D deep learning models with 3D input. We carry out a comprehensive comparison between the proposed method and these methods in classifying the ACA subtypes.

(1) Conventional classifiers. We obtain the Histogram of Oriented Gradients (HOG) feature, and five classic classifiers are employed to grade the ACA types: Support Vector Machine (SVM) (Suykens and Vandewalle, 1999), K-Nearest Neighbor (KNN) (Keller et al., 1985), AdaBoost (Li et al., 2008), Naive Bayes (McCallum and Nigam, 1998) and Random Forest (Pal, 
2005). In order to maintain consistency between comparable experiments, the HOG features are extracted from a $224 \times 224$ patch centered on the ACA region.

(2) Glaucoma classification models. Two specifically desgined ACA type classification networks are also compared in this work: Multi-Scale Regions Convolutional Neural Networks (MSCNN) (Hao et al., 2019), and Multi-Level Deep Network (MLDN) (Fu et al., 2019).

(3) 2D and 3D deep learning models. As our model uses the image sequence information, which may be considered to be $3 \mathrm{D}$ data, the following $2 \mathrm{D}$ and $3 \mathrm{D}$ architectures were used as baselines. 2D networks: AlexNet (Krizhevsky et al., 2012), VGG-16 (Simonyan and Zisserman, 2014), Inception-V3 (Xia et al., 2017), ResNet50 (He et al., 2016), and Xception (Chollet, 2017). 3D networks: C3D (Tran et al., 2015), I3D (Carreira and Zisserman, 2017), S3D (Xie et al., 2018), ResNet+ConvLSTM, and ResNet+ Bidirectional ConvLSTM (Bi-ConvLSTM) (Hanson et al., 2018).

It may be observed from TABLE 1 and TABLE 2 that the deep learning-based methods yield better classification performance than the HOG-based classifiers, since they can learn rich discriminative representations that are more powerful than visual features by using the multiple CNN layers. In the Dark and Bright datasets, the Xception model exhibits better performance than the other 2D models (with kappa score of 0.7248 and 0.7004 , respectively). A possible explanation is that its deeper architectures requires larger-scale datasets for effective fine-tuning.

By contrast, 3D networks achieves relatively higher performance than 2D networks. For example, the S3D model yielded kappas of 0.7431 and 0.7593 in the Dark and Bright datasets respectively. In addition, we also validate the ResNet+ConvLSTM and ResNet+Bi-ConvLSTM methods on dark, bright and merged dataset. The results show that the overall performances of the two methods are similar on three dataset and better than the other 3D networks. Therefore, considering the computational complexity and classification performance, we choose ResNet+ConvLSTM as the baseline of our proposed network. Overall, our MSDN outperforms the other networks in terms of all metrics by significant margins, and with kappas of 0.7920 and 0.7826 in the Dark and Bright datasets, respectively. The possible reason for this is that the proposed networks can learn rich and discriminative representation from both local features (2D image features) and global geometry (image sequence).

We also validate these methods on the merged dataset, which combines the Dark and Bright datasets into a single set. The results are illustrated in TABLE 2 . It can be clearly seen that all the networks demonstrate significant improvements in this dataset when compared with their classification performance using the Dark or Bright dataset alone. In addition, Fig. 8 shows the confusion matrices of ResNet-34, Xception, C3D, and our method over three datasets. These results further indicate the superiority of the performance on the merged dataset.

This scenario is consistent with the suggestion for AS-OCT screening in clinical practice (Xu et al., 2019): a more precise glaucoma type diagnosis can be achieved by using the AS-OCT data acquired under both dark and bright environments. A small ACA may be sufficient to open up an angle that would be closed under brighter illumination. This also suggests that the illumination conditions may be an important factor in the discrepancies between gonioscopic examination and AS-OCT screening.

\subsection{Ablation Study}

Our MSDN employs three blocks to form the classification framework: a ResNet-34 module, a ConvLSTM module and a carefully designed loss functions module -TC loss. To evaluate the effectiveness of each module, we report the results of different combinations of these modules over three datasets, and the results are reported in TABLE 3. They demonstrate that when applying the same modules to the Dark and Bright datasets, the classification results are relatively similar, and that classification on the merged dataset obtains the best performance overall.

To be more specific, it can be seen that the ResNet+ConvLSTM method clearly outperformed the ResNet alone, with improvement of about $10.39 \%$ and $11.42 \%$ in kappa over the Dark and Bright datasets, respectively. This shows 
Table 2: Different classification approaches for the grading of ACAs into open-, appositional-, and synechial-angles on merged datasets.

\begin{tabular}{|l||c|c|c|c|c|c|}
\hline \multicolumn{1}{|c||}{} & \multicolumn{6}{c|}{ Dark \& Bright Dataset } \\
\hline Methods & kappa & F1 & Acc & B-Acc & Se & Sp \\
\hline MLDN & 0.7210 & 0.7780 & 0.8861 & 0.8358 & 0.7700 & 0.9017 \\
MSDNN & 0.7249 & 0.7874 & 0.8868 & 0.8420 & 0.7797 & 0.9043 \\
Alexnet & 0.6869 & 0.7657 & 0.8697 & 0.8276 & 0.7617 & 0.8935 \\
VGG-16 & 0.7100 & 0.7710 & 0.8814 & 0.8313 & 0.7641 & 0.8985 \\
Resnet-34 & 0.7067 & 0.7658 & 0.8804 & 0.8281 & 0.7593 & 0.8970 \\
Inception-V3 & 0.7386 & 0.8008 & 0.8919 & 0.8512 & 0.7931 & 0.9094 \\
Xception & 0.7352 & 0.7907 & 0.8917 & 0.8440 & 0.7814 & 0.9065 \\
\hline C3D & 0.7770 & 0.8300 & 0.9078 & 0.8710 & 0.8200 & 0.9219 \\
I3D & 0.7777 & 0.8244 & 0.9091 & 0.8657 & 0.8111 & 0.9203 \\
S3D & 0.7607 & 0.8062 & 0.9028 & 0.8537 & 0.7937 & 0.9137 \\
\hline ResNet+ConvLSTM & 0.8025 & 0.8536 & 0.9175 & 0.8895 & 0.8470 & 0.9320 \\
ResNet+Bi-ConvLSTM & 0.8037 & 0.8547 & 0.9179 & 0.8895 & 0.8470 & 0.9320 \\
\hline MSDN & $\mathbf{0 . 8 0 9 8}$ & $\mathbf{0 . 8 5 5 1}$ & $\mathbf{0 . 9 2 1 3}$ & $\mathbf{0 . 8 8 7 7}$ & $\mathbf{0 . 8 4 3 0}$ & $\mathbf{0 . 9 3 2 4}$ \\
\hline
\end{tabular}

that the ConvLSTM learn discriminative representations from an AS-OCT image sequence, and is capable of preserving the spatial information, so memorizing the change in appearance that corresponds to motion information (neighboring B-scans), thereby improving performance in separating appositional angle and fully closed angle.

TABLE 3 also reveals that after TC loss is applied, giving the combination (ResNet+ConvLSTM+TC), classification performance improves significantly, with an improvement of approximately $2.31 \%,-0.94 \%$, and $0.73 \%$ in kappa over the Dark, Bright, and merged datasets, respectively, when compared with the ResNet+ConvLSTM method. The possible reason is that with the TC loss, the ConvLSTM explores the spatial state of appearance features of a AS-OCT sequence, and these features are further aggregated for classification purposes.

\section{Discussion}

In this subsection, we analyze and discuss the network under different scenarios: the effectiveness of different size of ACA region and the effectiveness of image alignment.

\subsection{The effectiveness of image alignment}

We report the results obtained in the absence of the the image alignment step (MSDN w/o alignment). As may be observed, image alignment clearly assists classification in yielding higher performance in terms of all the metrics by significant margins, with improvements of $0.82 \%, 1.62 \%$, and $4.15 \%$ in kappa, sensitivity, and specificity over the Dark dataset, respectively, when compared with the direct application of the proposed network to the original images (w/o alignment). This finding shows that image alignment is a crucial preprocessing step in improving the accuracy of subsequent classification.

\subsection{The effectiveness of the size of the ACA region}

Choosing a suitable ACA region size is usually empiric and case-specific for most angle-closure detection methods (Fu et al., 2019, 2018; Xu et al., 2013). On one hand, too large the ACA size leads to over-intensive computation. On the other hand, too small an ACA region results in loss of the appearance features. In the experiment, we compared two sizes of ACA regions: $224 \times 224$, and $448 \times 448$ pixels. As may be observed in TABLE 3, methods using ACA region of $224 \times 224$ pixels performed better than those using ACA regions of $448 \times 448$ pixels. This implies that when sized of $224 \times 224$ pixels, the ACA patch focuses on the most highly relevant details, such as the iris and trabecular. By contrast, when the ACA patch is enlarged to $448 \times 448$ pixels, the larger region may contain more unrelated local features, which may disturb the deep network.

\subsection{Classification of appositional-and synechial-angle}

As we claimed in Section I, the classification of ACAs into two subtypes, i.e., open angle and angle-closure, is relatively straightforward and easy, and recent works have demonstrated 

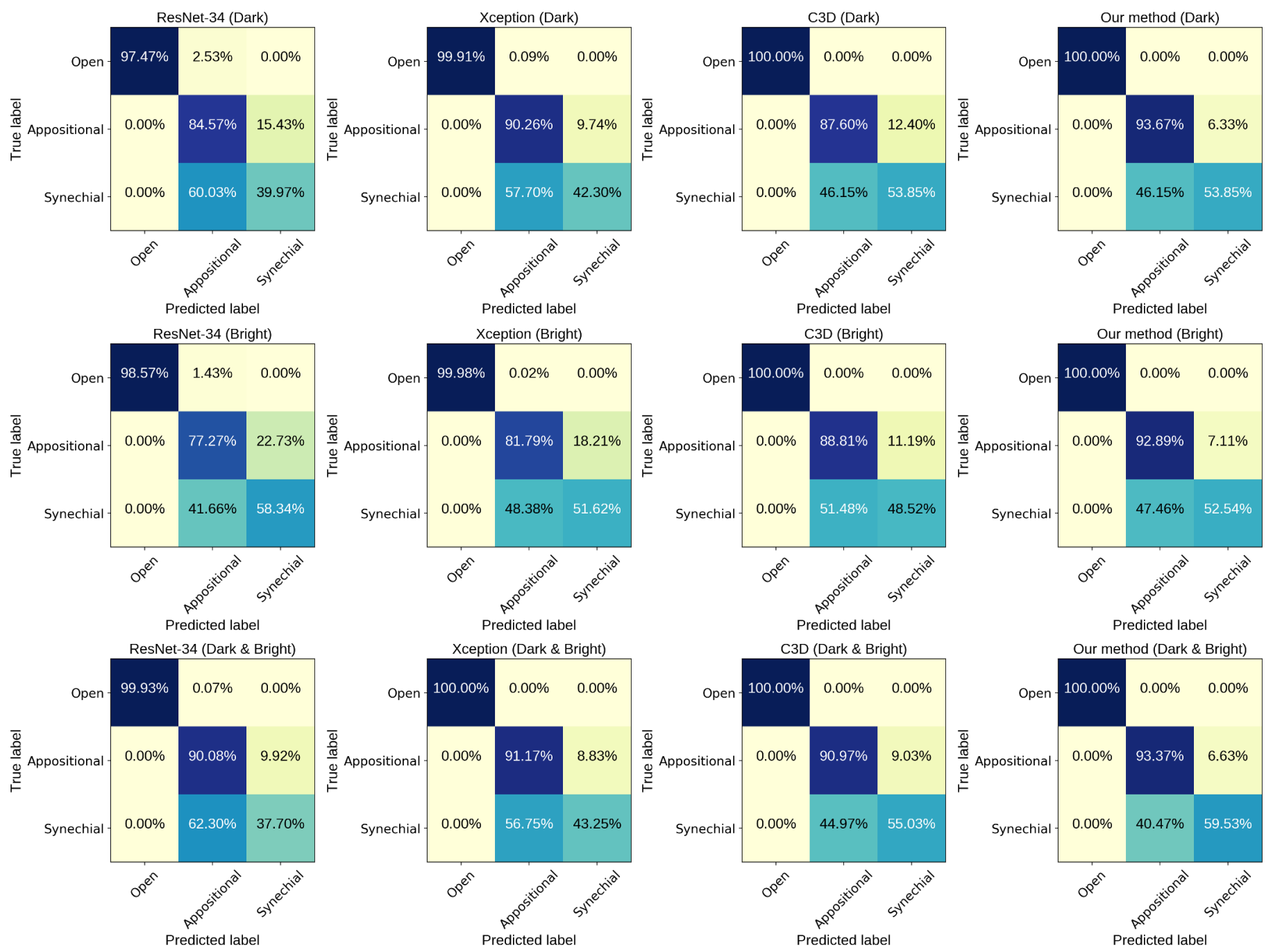

Fig. 8: Confusion matrices of some state-of-the-art 2D and 3D deep networks and our method in classifying appositional, and synechial ACA over three AS-OCT datasets. The numbers in the confusion matrices denote the percentage of the predicted class.

remarkable performance (AUC $\geq 0.98$ ). In our experiment, according to confusion matrices in Fig. 8, we also receive an $\mathrm{ACC}=1.000$ using the proposed MSDN for open angle and angle-closure classification, and the other state-of-the-art networks also obtained similar performance. Nevertheless, we intend in this subsection to report only the performance in classification of appositional and synechial angles.

Fig. 9 demonstrates the classification performance of five classic conventional classifiers. It can be seen clearly that these classifiers exhibit poor performance in separating appositionaland synechial- angles, and the Naive Bayes classifier perform the best in these classifiers, where AUCs are only of 0.6183 , 0.6648 , and 0.6376, respectively over the Dark, Bright, and merged datasets.

By contrast, deep neural networks obtained much higher AUC scores than the conventional classifiers over all the datasets, as shown in Fig. 10. However, we can observe that the proposed method still produces the best performance on the Dark, Bright, and merged datasets with AUCs of 0.8158, 0.8005 , and 0.8444 . In addition, most of the deep learningbased approaches are able to generate the higher classification results on merged dataset, and this finding tends to confirm that the use of images captured under both dark and bright illumination conditions could improve the diagnostic accuracy.

Fig. 11 reveals the classification performance in distinguishing appositional and synechial angles under different scenar- 
Table 3: Performance of different module combinations for the grading of ACAs into open-, appositional-, and synechial-angles.

\begin{tabular}{|l||c|c|c|c||c|c|c|c||c|c|c|c|}
\hline \multicolumn{1}{|c||}{} & \multicolumn{4}{|c||}{ Dark Dataset } & \multicolumn{4}{c||}{ Bright Dataset } & \multicolumn{3}{c|}{ Dark \& Bright Dataset } \\
\hline Methods & kappa & F1 & Se & Sp & kappa & F1 & Se & Sp & kappa & F1 & Se & Sp \\
\hline ResNet $(448 \times 448)$ & 0.7042 & 0.7660 & 0.7596 & 0.8965 & 0.6901 & 0.7530 & 0.7481 & 0.8917 & 0.7114 & 0.7730 & 0.7669 & 0.8979 \\
ResNet $(224 \times 224)$ & 0.6650 & 0.7484 & 0.7401 & 0.8878 & 0.6778 & 0.7786 & 0.7806 & 0.9011 & 0.7067 & 0.7658 & 0.7593 & 0.8970 \\
ResNet+ConLSTM $(448 \times 448)$ & 0.7629 & 0.8256 & 0.8215 & 0.9195 & 0.7496 & 0.8092 & 0.8009 & 0.9130 & 0.7779 & 0.8347 & 0.8281 & 0.9237 \\
ResNet+ConvLSTM $(224 \times 224)$ & 0.7689 & 0.8281 & 0.8220 & 0.9208 & 0.7920 & 0.8450 & 0.8377 & 0.9282 & 0.8025 & 0.8536 & 0.8470 & 0.9320 \\
ResNet+ConvLSTM+TC $(448 \times 448)$ & 0.7730 & 0.8291 & 0.8210 & 0.9213 & 0.7747 & 0.8310 & 0.8236 & 0.9221 & 0.8045 & 0.8508 & 0.8386 & 0.9306 \\
ResNet+ConvLSTM+TC $(224 \times 224)$ & 0.7920 & 0.8382 & 0.8250 & 0.9257 & 0.7826 & 0.8304 & 0.8181 & 0.9227 & 0.8098 & 0.8551 & 0.8430 & 0.9324 \\
\hline MSDN $(448 \times 448)$ w/o alignment & 0.7697 & 0.8240 & 0.8142 & 0.9194 & 0.7667 & 0.8218 & 0.8123 & 0.9185 & 0.7825 & 0.8361 & 0.8326 & 0.9267 \\
ResNet+ConLLSTM+TC $(448 \times 448)$ & 0.7730 & 0.8291 & 0.8210 & 0.9213 & 0.7744 & 0.8310 & 0.8236 & 0.9221 & 0.8045 & 0.8508 & 0.8386 & 0.9306 \\
MSDN (224 $\times$ 224) w/o alignment & 0.7838 & 0.8342 & 0.8231 & 0.9238 & 0.7664 & 0.8152 & 0.8030 & 0.9166 & 0.7683 & 0.8321 & 0.8239 & 0.9183 \\
ResNet+ConvLSTM+TC $(224 \times 224)$ & 0.7920 & 0.8382 & 0.8250 & 0.9257 & 0.7826 & 0.8304 & 0.8181 & 0.9227 & 0.8098 & 0.8551 & 0.8430 & 0.9324 \\
\hline
\end{tabular}

Dark

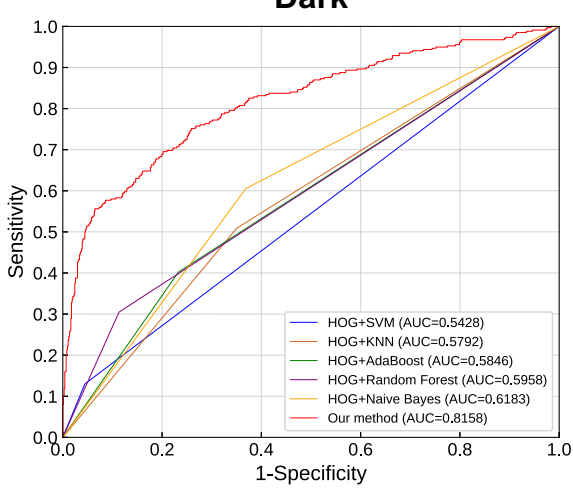

Bright

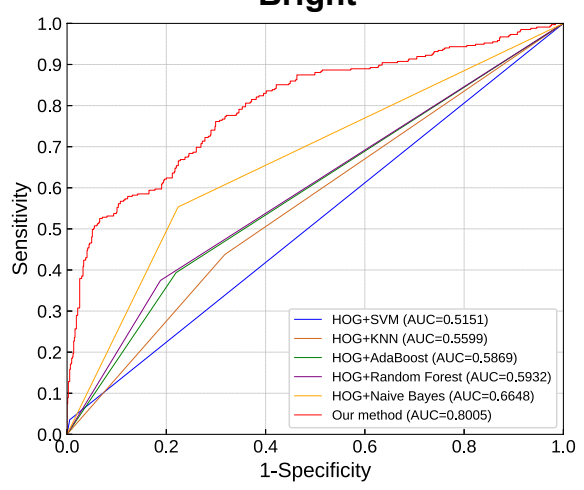

Dark \& Bright

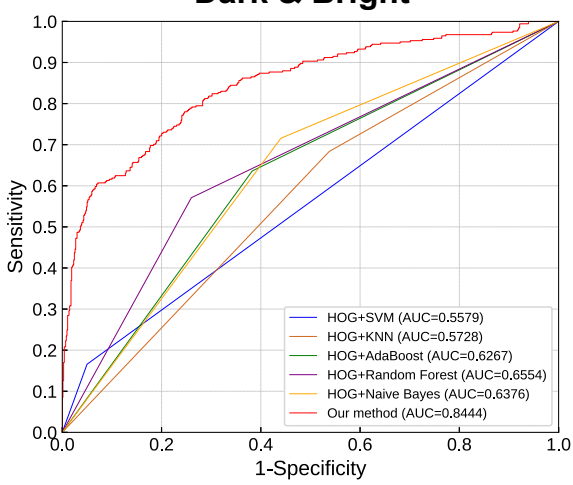

Fig. 9: Comparison of conventional classifiers and our method in classifying appositional, and synechial ACA over three AS-OCT datasets.

ios: different module combinations, different ACA region sizes, and with and without image alignment. The results demonstrate that the combination of the proposed network architecture (ResNet+ConvLSTM+TC), the application of image alignment, and the selection of an ACA region with size of $224 \times 224$ pixels, are the important factors in obtaining better classification results of appositional and synechial angles.

\section{Conclusion}

Various methods have recently been proposed to identify gonioscopic angle-closure from AS-OCT imaging. However, most of the existing automated methods are only able to classify the anterior chamber angles as either open or angle-closure. In this paper, we have proposed an image sequence-based deep network that is able to classify the anterior chamber angles in AS-OCT imagery into three types - open-angle, appositionalangle, and synechial-angle, so as to further guide clinical man- agement at different stages of glaucoma.

The proposed method consists of image alignment, iris segmentation, ACA localization and ACA classification. In the deep learning part, we have proposed a novel loss - TC loss with a view to learning discriminative representations over two different illumination condition datasets. We have reported that the proposed method is able to accurately classify ACAs into our three groups, with an Acc of 0.9146, when compared with the gonioscopic annotation. The results show that our method achieves better performance when compared with other stateof-the-art 2D and 3D deep networks.

We have constructed an AS-OCT dataset containing images from 66 eyes, and the AS-OCT of these eyes were acquired under both dark and bright lightness conditions. Such data construction is potentially able to simulate the pressure of the goniolens, which can push the angle open and help determine the true angle configuration. It may be expected that the ability of 
Dark

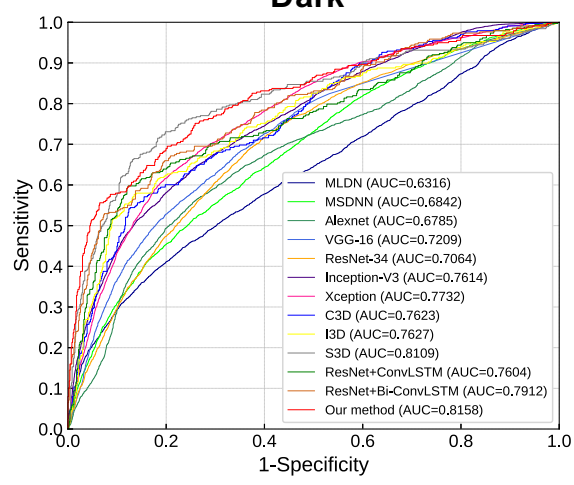

Bright

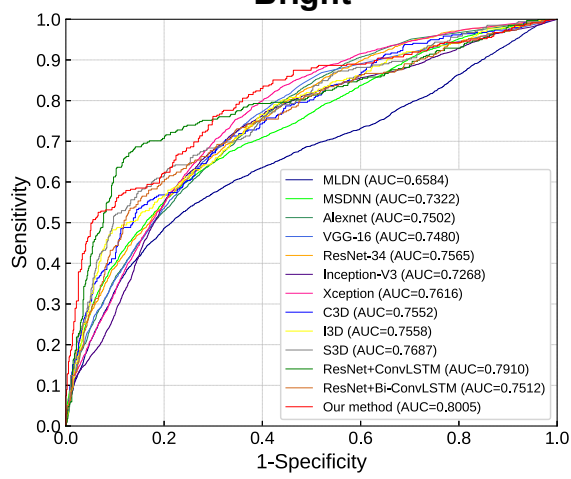

Dark \& Bright

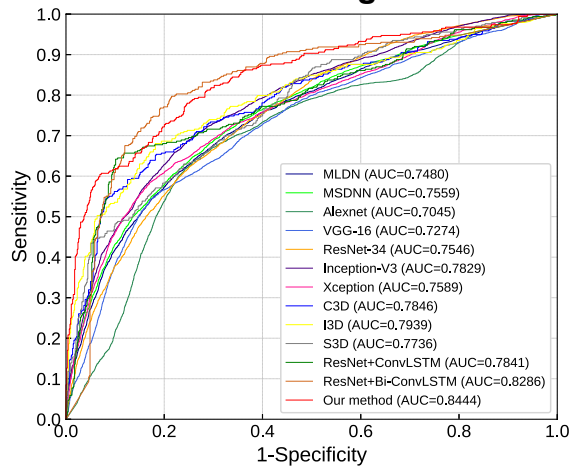

Fig. 10: Comparison of state-of-the-art 2D and 3D deep networks and our method in classifying appositional, and synechial ACA over three AS-OCT datasets.

Dark

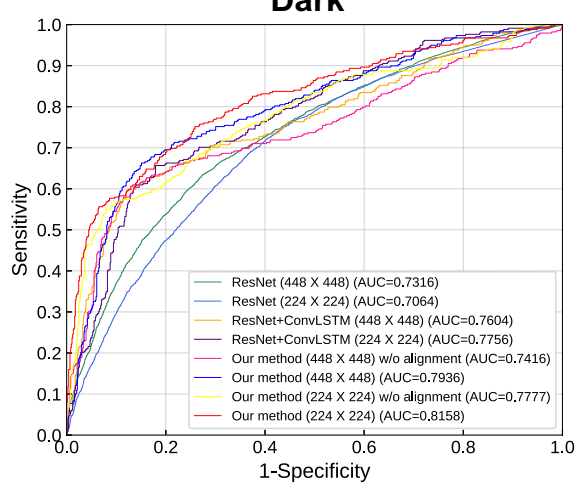

Bright

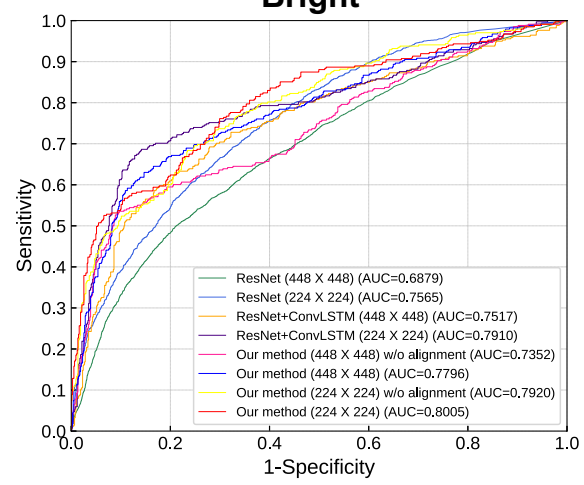

Dark \& Bright

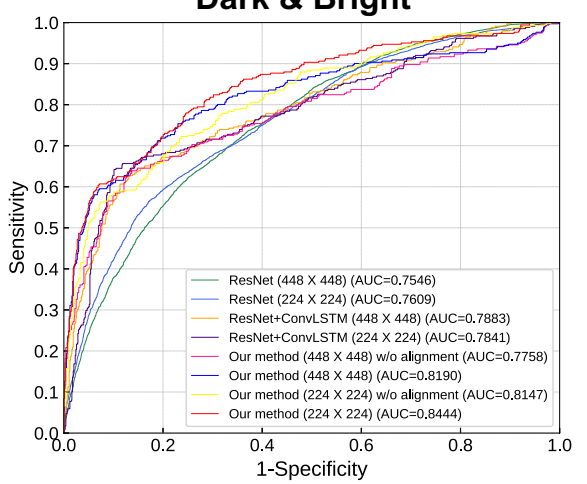

Fig. 11: Classification of appositional and synechial angles under different scenarios: different module combination, different ACA region size, and with and without image alignment over three AS-OCT datasets.

the proposed model to learn discriminating features from these datasets could be a powerful tool for diagnosing the presence, and analyzing the progression of angle-closure diseases. In addition, we believe this work has the potential to be applied to other 3D image modalities such as MRI, CT and OCT, which however is not the focus of the paper. In the future, we will try to extend the proposed method to other medical image datasets to solve similar problems.

\section{Acknowledgement}

This work was supported by Zhejiang Provincial Natural Science Foundation of China (LZ19F010001, LQ19H180001), the Key Research and Development Program of Zhejiang Province (2020C03036), Ningbo '2025 S\&T Megaprojects' (2019B10033, 2019B10061).

\section{References}

Amil, P., et al., 2019. Unsupervised feature extraction of anterior chamber oct images for ordering and classification. Scientific reports 9, 1157.

Aptel, F., Chiquet, C., Beccat, S., Denis, P., 2012. Biometric evaluation of anterior chamber changes after physiologic pupil dilation using pentacam and anterior segment optical coherence tomography. Investigative ophthalmology \& visual science 53, 4005-4010.

Badrinarayanan, V., Kendall, A., Cipolla, R., 2017. Segnet: A deep convolutional encoder-decoder architecture for image segmentation. IEEE Trans. Pattern Anal. Mach. Intell. 39, 2481-2495.

Carreira, J., Zisserman, A., 2017. Quo vadis, action recognition? a new model and the kinetics dataset, in: proceedings of CVPR, pp. 6299-6308.

Casson, R., Newland, H., Muecke, J., McGovern, S., Abraham, L., Shein, W., Selva, D., Aung, T., 2007. Gonioscopy findings and prevalence of occludable angles in a burmese population: the meiktila eye study. British journal of ophthalmology 91, 856-859.

Cheng, J., et al., 2016. Speckle reduction in 3d optical coherence tomography of retina by a-scan reconstruction. IEEE Trans. Med. Imaging 35, 2270-2279.

Chollet, F., 2017. Xception: Deep learning with depthwise separable convolutions, in: Proceedings of CVPR, pp. 1251-1258.

Console, J.W., Sakata, L.M., Aung, T., Friedman, D.S., He, M., 2008. Quantitative analysis of anterior segment optical coherence tomography images: the zhongshan angle assessment program. Br. J. Ophthalmol. 92, 1612-1616.

Foster, P., Aung, T., Nolan, W., Machin, D., Baasanhu, J., Khaw, P., Alsbirk, P., Lee, P., Seah, S., Johnson, G., 2004. Defining "occludable" angles in population surveys: drainage angle width, peripheral anterior synechiae, and glaucomatous optic neuropathy in east asian people. British Journal of Ophthalmology $88,486-490$. 
Foster, P.J., Buhrmann, R., Quigley, H.A., Johnson, G.J., 2002. The definition and classification of glaucoma in prevalence surveys. British journal of ophthalmology 86, 238-242.

Foster, P.J., Johnson, G.J., 2001. Glaucoma in china: how big is the problem? Br. J. Ophthalmol. 85, 1277-1282.

Fu, H., Li, F., Sun, X., Cao, X., Liao, J., Orlando, J.I., Tao, X., Li, Y., Zhang, S., Tan, M., et al., 2020. Age challenge: Angle closure glaucoma evaluation in anterior segment optical coherence tomography. arXiv preprint arXiv:2005.02258.

Fu, H., et al., 2017. Segmentation and quantification for angle-closure glaucoma assessment in anterior segment oct. IEEE Trans. Med. Imaging 36, 1930-1938.

Fu, H., et al., 2018. Multi-context deep network for angle-closure glaucoma screening in anterior segment oct, in: MICCAI, pp. 356-363.

$\mathrm{Fu}, \mathrm{H}$., et al., 2019. Angle-closure detection in anterior segment oct based on multilevel deep network. IEEE transactions on cybernetics .

Gazzard, G., Foster, P.J., Friedman, D.S., Khaw, P., Seah, S., 2004. Light to dark physiological variation in irido-trabecular angle width. Br J Ophthalmol 88, 1357-1482.

Girshick, R., 2015. Fast r-cnn, in: Proceedings of ICCV, pp. 1440-1448.

Girshick, R., Donahue, J., Darrell, T., Malik, J., 2014. Rich feature hierarchies for accurate object detection and semantic segmentation, in: Proceedings of CVPR, pp. 580-587.

Greff, K., Srivastava, R.K., Koutník, J., Steunebrink, B.R., Schmidhuber, J., 2016. Lstm: A search space odyssey. IEEE transactions on neural networks and learning systems 28, 2222-2232.

$\mathrm{Gu}, \mathrm{Z}$., et al., 2019. Ce-net: Context encoder network for $2 \mathrm{~d}$ medical image segmentation. IEEE Trans. Med. Imaging .

Hanson, A., Pnvr, K., Krishnagopal, S., Davis, L., 2018. Bidirectional convolutional lstm for the detection of violence in videos, in: Proceedings of the European Conference on Computer Vision (ECCV), pp. 0-0.

Hao, H., et al., 2019. Anterior chamber angles classification in anterior segment oct images via multi-scale regions convolutional neural networks, in: EMBC, IEEE. pp. 849-852.

He, K., Zhang, X., Ren, S., Sun, J., 2016. Deep residual learning for image recognition, in: Proceedings of CVPR, pp. 770-778.

He, M., Foster, P.J., Ge, J., Huang, W., Wang, D., Friedman, D.S., Khaw, P.T., 2006. Gonioscopy in adult chinese: the liwan eye study. Investigative ophthalmology \& visual science 47, 4772-4779.

Hirose, F., Hata, M., Ito, S.i., Matsuki, T., Kurimoto, Y., 2013. Light-dark changes in iris thickness and anterior chamber angle width in eyes with occludable angles. Graefe's Archive for Clinical and Experimental Ophthalmology 251, 2395-2402.

Keller, J.M., Gray, M.R., Givens, J.A., 1985. A fuzzy k-nearest neighbor algorithm. IEEE transactions on systems, man, and cybernetics , 580-585.

Krizhevsky, A., Sutskever, I., Hinton, G.E., 2012. Imagenet classification with deep convolutional neural networks, in: Proceedings of NIPS, pp. 10971105 .

Lee, R.Y., Lin, S.C., Chen, R.I., Barbosa, D.T., Lin, S.C., 2016. Association between light-to-dark changes in angle width and iris parameters in light, dark and changes from light-to-dark conditions. British Journal of Ophthalmology 100, 1274-1279.

Li, X., Wang, L., Sung, E., 2008. Adaboost with svm-based component classifiers. Engineering Applications of Artificial Intelligence 21, 785-795.

Malhotra, P., Ramakrishnan, A., Anand, G., Vig, L., Agarwal, P., Shroff, G., 2016. Lstm-based encoder-decoder for multi-sensor anomaly detection. arXiv preprint arXiv:1607.00148 .

Matsuo, M., Pajaro, S., De Giusti, A., Tanito, M., 2020. Automated anterior chamber angle pigmentation analyses using $360^{\circ}$ gonioscopy. British Journal of Ophthalmology 104, 636-641.

McCallum, A., Nigam, K., 1998. A comparison of event models for naive bayes text classification, in: AAAI-98 workshop on learning for text categorization, Citeseer. pp. 41-48.

Ni Ni, S., Tian, J., Marziliano, P., Wong, H.T., 2014. Anterior chamber angle shape analysis and classification of glaucoma in ss-oct images. J. Ophthalmol. 2014.

Nolan, W.P., See, J.L., Chew, P.T., Friedman, D.S., Smith, S.D., Radhakrishnan, S., Zheng, C., Foster, P.J., Aung, T., 2007. Detection of primary angle closure using anterior segment optical coherence tomography in asian eyes. Ophthalmology 114, 33-39.

Pal, M., 2005. Random forest classifier for remote sensing classification. International Journal of Remote Sensing 26, 217-222.
Radhakrishnan, S., Huang, D., Smith, S.D., 2005. Optical coherence tomography imaging of the anterior chamber angle. Ophthalmology Clinics of North America 18, 375-81.

Redmon, J., Divvala, S., Girshick, R., Farhadi, A., 2016. You only look once: Unified, real-time object detection, in: Proceedings of CVPR, pp. 779-788.

Ren, S., He, K., Girshick, R., Sun, J., 2015. Faster r-cnn: Towards real-time object detection with region proposal networks, in: Proceedings of NIPS, pp. 91-99.

Ronneberger, O., Fischer, P., Brox, T., 2015. U-net: Convolutional networks for biomedical image segmentation, in: MICCAI, pp. 234-241.

Sawaguchi, S., et al., 2012. Prevalence of primary angle closure and primary angle-closure glaucoma in a southwestern rural population of japan: the kumejima study. Ophthalmology 119, 1134-1142.

Shang, Q., et al., 2019. Automated iris segmentation from anterior segment oct images with occludable angles via local phase tensor, in: EMBC, IEEE. pp. 4745-4749.

Simonyan, K., Zisserman, A., 2014. Very deep convolutional networks for large-scale image recognition. arXiv preprint arXiv:1409.1556

Suykens, J.A., Vandewalle, J., 1999. Least squares support vector machine classifiers. Neural processing letters 9, 293-300.

Tham, Y.C., Li, X., Wong, T.Y., Quigley, H.A., Aung, T., Cheng, C.Y., 2014. Global prevalence of glaucoma and projections of glaucoma burden through 2040: a systematic review and meta-analysis. Ophthalmology 121, 20812090.

Tian, J., Marziliano, P., Baskaran, M., Wong, H.T., Aung, T., 2011. Automatic anterior chamber angle assessment for hd-oct images. IEEE Trans. Biomed. Eng 58, 3242-3249.

Tran, D., Bourdev, L., Fergus, R., Torresani, L., Paluri, M., 2015. Learning spatiotemporal features with $3 \mathrm{~d}$ convolutional networks, in: Proceedings of ICCV, pp. 4489-4497.

Williams, D., et al., 2016. Reconstruction of $3 \mathrm{~d}$ surface maps from anterior segment opticalcoherence tomography images using graph theory and geneticalgorithms. Biomedical Signal Processing and Control 25, 91-98.

Xia, X., Xu, C., Nan, B., 2017. Inception-v3 for flower classification, in: ICIVC, IEEE. pp. 783-787.

Xie, S., Sun, C., Huang, J., Tu, Z., Murphy, K., 2018. Rethinking spatiotemporal feature learning: Speed-accuracy trade-offs in video classification, in: Proceedings of ECCV, pp. 305-321.

Xingjian, S., Chen, Z., Wang, H., Yeung, D.Y., Wong, W.K., Woo, W.c., 2015. Convolutional lstm network: A machine learning approach for precipitation nowcasting, in: Proceedings of NIPS, pp. 802-810.

Xu, B.Y., Chiang, M., Chaudhary, S., Kulkarni, S., Pardeshi, A.A., Varma, R., 2019. Deep learning classifiers for automated detection of gonioscopic angle closure based on anterior segment oct images. Am. J. Ophthalmol.

$\mathrm{Xu}$, Y., et al., 2012. Anterior chamber angle classification using multiscale histograms of oriented gradients for glaucoma subtype identification, in: EMBC, IEEE. pp. 3167-3170.

$\mathrm{Xu}, \mathrm{Y}$., et al., 2013. Automated anterior chamber angle localization and glaucoma type classification in oct images, in: EMBC, IEEE. pp. 7380-7383.

Zhang, Z., et al., 2019. Gait recognition via disentangled representation learning, in: Proceedings of CVPR, pp. 4710-4719.

Zheng, S., et al., 2015. Conditional random fields as recurrent neural networks, in: Proceedings of ICCV, pp. 1529-1537.

Zhu, S., Ma, K.K., 2000. A new diamond search algorithm for fast blockmatching motion estimation. IEEE Trans. Med. Imaging 9, 287-290. 
2021-01-07

\section{Angle-closure assessment in anterior segment OCT images via deep learning}

Hao, Huaying

Elsevier

Hao H, Zhao Y, Yan Q, et al., (2021) Angle-closure assessment in anterior segment OCT

images via deep learning. Medical Image Analysis, Volume 69, April 2021, Article number 101956

https://doi.org/10.1016/j.media.2021.101956

Downloaded from Cranfield Library Services E-Repository 